\title{
Witnessing Dp-Rank
}

\author{
Itay Kaplan and Pierre Simon
}

\begin{abstract}
We prove that in $\mathrm{NTP}_{2}$ theories the dp-rank of a type can be witnessed by indiscernible sequences of tuples satisfying that type. If the type has dp-rank infinity, then this can be witnessed by singletons (in any theory).
\end{abstract}

\section{Introduction}

In this note we answer a question of Alf Onshuus and Alexander Usvyatsov, whether dp-minimality can be witnessed by indiscernible sequences of singletons. We prove two general theorems regarding dp-rank.

Let Card denote the class of cardinals. We define Card* to be the class Card to which we add an element $\kappa_{-}$for each infinite cardinal $\kappa$. We extend the linear order from Card to Card* by setting $\mu<\kappa_{-}<\kappa$ whenever $\mu<\kappa$ are cardinals.

Definition 1.1 Let $p(x)$ be a partial (consistent) type over a set $A$ ( $x$ is a finite tuple, here and throughout the paper). We define the $d p$-rank of $p(x)$ (which is an element of Card* or $\infty$ ) as follows.

- Let $\kappa$ be a cardinal. We say that $p(x)$ has dp-rank $<\kappa$ (which we write rk-dp $(p)<\kappa)$ if given any realization $a$ of $p$ and any $\kappa$ mutually indiscernible sequences over $A$, at least one of them is indiscernible over $A a$.

- We say that $p$ has dp-rank $\kappa$ over $A$ (or $\operatorname{rk}-\operatorname{dp}(p)=\kappa)$ if it has dp-rank $<\mu$ for all $\mu>\kappa$, but it is not the case that $\operatorname{rk}-\operatorname{dp}(p)<\kappa$.

- If $\kappa$ is an infinite cardinal, we say that $p$ has dp-rank $\kappa_{-}$over $A$ (or rk-dp $\left.(p)=\kappa_{-}\right)$if it has dp-rank $<\kappa$, but for no $\mu<\kappa$ do we have $\operatorname{rk}-\operatorname{dp}(p)<\mu$.

- If rk-dp $(p)<\kappa$ holds for no cardinal $\kappa$, then we say that $p$ has dp-rank $\infty$.

- We call $p$ dp-minimal if it has dp-rank 1 .

- We call $p$ dependent if $\operatorname{rk}-\operatorname{dp}(p)<\infty$. This is equivalent to $\operatorname{rk}-\operatorname{dp}(p)<|T|^{+}$ (see Corollary 2.3).

Received January 27, 2012; accepted October 26, 2012

2010 Mathematics Subject Classification: Primary 03C45; Secondary 03C68

Keywords: NIP, dp-rank

(C) 2014 by University of Notre Dame 10.1215/00294527-2688105 
Remark 1.2 It is easy to see that the set $A$ does not matter, as long as $p$ is defined over it. Indeed, for a set $B$ over which $p$ is defined, let us define for the sake of discussion $\operatorname{rk}-\operatorname{dp}(p, B)$ as the dp-rank of $p$ over $B$ similarly to the definition above; but we add the requirement that the sequences are mutually indiscernible over $B$. If $A \subseteq B$, and $p$ is a type over $A$, then it is easy to see that $\operatorname{rk-dp}(p, B) \leq \operatorname{rk}-\operatorname{dp}(p, A)$, while the other direction uses a standard application of Ramsey theorem, so rk-dp $(p, B)=\operatorname{rk}-\operatorname{dp}(p, A)$.

Note also that if $q(x)$ extends $p(x)$, then $\operatorname{rk}-\operatorname{dp}(p(x)) \geq \operatorname{rk}-\operatorname{dp}(q(x))$, so we have the following.

Remark 1.3 Any extension of a dependent type is dependent.

Recall the following.

Definition 1.4 A (complete, first-order) theory $T$ is $d p$-minimal if the type $\{x=x\}$ is dp-minimal. The theory $T$ is dependent if the type $\{x=x\}$ is dependent.

Dp-rank and dependent types were originally defined by Usvyatsov in [7] and further studied by Onshuus in [4]. Dp-rank is a simplification of the various ranks appearing in Shelah [5]. We use a slightly different convention for it than those two papers, which has the advantage of distinguishing between $\kappa$ and $\kappa_{-}$. Yet another convention is used in Kaplan, Onshuus, and Usvyatsov [3], which has the disadvantage of giving a different meaning to $\operatorname{rk}-\operatorname{dp}(p)=\kappa$ depending on whether $\kappa$ is finite or infinite. Dp-minimality was first defined in [4]. It is shown in Simon [6] that the original definition of dp-minimality is equivalent to the definition given here.

Examples of dp-minimal theories include all o-minimal theories and C-minimal theories.

Note that the sequences that witness $\operatorname{rk}-\operatorname{dp}(p) \geq \kappa$ in Definition 1.1 can always be taken to be sequences of finite tuples, but can we bound the length?

Question (A. Onshuus, A. Usvyatsov) Can we assume in the definition of dpminimality that the indiscernible sequences are sequences of singletons?

We provide a positive answer in Corollary 1.7 below, but we need to add parameters to the base.

We prove the following two theorems.

Main Theorem A If $p$ is a type over $A$ which is independent (i.e., $\operatorname{rk}-\mathrm{dp}(p)=\infty$ ), then there is some $A^{\prime} \supseteq A$ such that $\left|A^{\prime} \backslash A\right|$ is finite, a realization $a \models p$ and $A^{\prime}$ mutually indiscernible sequences of singletons $\left\langle\left. I_{i}|i<| T\right|^{+}+|A|^{+}\right\rangle$such that $I_{i}$ is not indiscernible over $A^{\prime}$ a for all $i$.

From this we will deduce the following.

Corollary 1.5 To check whether a theory is dependent it is enough to check that for every indiscernible sequence of singletons $\left\langle\left. a_{i}|i<| T\right|^{+}\right\rangle$over some finite A, and for every singleton $c$, there is $\alpha<|T|^{+}$such that $\left\langle a_{i} \mid i>\alpha\right\rangle$ is indiscernible over Ac.

The second result is about dependent types, but to prove it we need to assume ${ }^{1}$ that the theory is $\mathrm{NTP}_{2}$. 
Definition 1.6 A theory $T$ is $\mathrm{NTP}_{2}$ (does not have the tree property of the second kind) if there is no formula $\varphi(x, y)$ and array $\left\langle a_{i, j} \mid i, j<\omega\right\rangle$ such that for every $i<\omega,\left\{\varphi\left(x, a_{i, j}\right) \mid j<\omega\right\}$ is $k$-inconsistent (i.e., each subset of size $k$ is inconsistent) and for every $\eta: \omega \rightarrow \omega$, the set $\left\{\varphi\left(x, a_{i, \eta(i)}\right) \mid i<\omega\right\}$ is consistent.

The class of $\mathrm{NTP}_{2}$ theories contains both simple and dependent theories.

Main Theorem B Assume that $T$ is $\mathrm{NTP}_{2}$ and that $p$ is a dependent type over $A$ with $\operatorname{rk}-\operatorname{dp}(p) \geq \kappa$. Then there are some $A^{\prime} \supseteq A$, some $a \models p$, and $A^{\prime}$-mutually indiscernible sequences $\left\{I_{i} \mid i<\kappa\right\}$ such that each of them is not indiscernible over $A^{\prime} a$ and all tuples in each $I_{i}$ satisfy $p$.

Note that we may always choose $A^{\prime}$ so that $\left|A^{\prime} \backslash A\right|$ is at most $\kappa+\boldsymbol{\aleph}_{0}$ since, for each sequence $I_{i}$, we only need finitely many parameters from $A^{\prime}$ to witness that $I_{i}$ is not indiscernible over $A^{\prime} a$.

Now we can answer Question 1.

Corollary 1.7 If $T$ is not dp-minimal, then there is some finite set $A^{\prime}$, some singleton $a$, and two $A^{\prime}$-mutually indiscernible sequences $\{I, J\}$ of singletons such that both $I$ and $J$ are not indiscernible over $A^{\prime} a$.

Proof Right to left is obvious. For the other direction, if $T$ is dependent, then we may use Main Theorem B. (Since there are only two sequences, only finitely many parameters from $A^{\prime}$ are needed to witness nonindiscernibility, so we may assume that $A^{\prime}$ is finite.) But if $T$ is not dependent, then by Main Theorem A there exist such $a$, $A$, and infinitely many such sequences.

The following question remains open.

Question 1.8 (J. Ramakrishnan) Can we assume in the definition of dp-rank that the indiscernible sequences are sequences of singletons by adding parameters to the base?

Our results show that this is indeed the case when the type is independent or when it is the type of a singleton in an $\mathrm{NTP}_{2}$-theory.

In Section 2 we prove Main Theorem A, and in Section 3 we prove Main Theorem B.

Question 1.9 Are the extra parameters in the main theorems needed?

Throughout the paper, $\mathfrak{C}$ will denote a monster model of the theory $T$ (i.e., a very big saturated model).

\section{On Dependent Types and a Proof of Main Theorem A}

2.1 On dependent types We start with the following easy observation (which is somewhat similar to [4, Observation 2.7]), with a very straightforward proof.

Claim 2.1 Suppose that $p(x)$ is a partial type over A. Then the following are equivalent:

1. there are $a \models p$ and A-mutually indiscernible sequences $\left\langle I_{i} \mid i<\omega\right\rangle$ such that the sequence $\left\langle I_{i} \mid i<\omega\right\rangle$ is indiscernible over Aa, and for each $i, I_{i}$ is not indiscernible over Aa;

2. $p$ is independent; 
3. $\operatorname{rk}-\mathrm{dp}(p) \geq|T|^{+}+|A|^{+}$;

4. there is an A-indiscernible sequence $\left\langle a_{i} \mid i<\omega\right\rangle$ such that $a_{i} \models p, a$ formula $\varphi(x, y)$, and some $c$ such that $\varphi\left(a_{i}, c\right)$ holds iff $i$ is even;

5. there is an A-indiscernible sequence $\left\langle b_{i} \mid i<\omega\right\rangle$, a formula $\psi(y, x)$, and some $d \models p$ such that $\psi\left(b_{i}, d\right)$ holds iff $i$ is even;

6. there is a set $\left\{a_{i} \mid i<\omega\right\}$ of realizations of $p$ and a formula $\varphi(x, y)$ such that for every $s \subseteq \omega$, there is some $c_{s}$ such that $\varphi\left(a_{i}, c_{s}\right)$ holds iff $i \in s$;

7. there is a set $\left\{b_{i} \mid i<\omega\right\}$ and a formula $\psi(y, x)$ such that for every $s \subseteq \omega$, there is some $d_{s} \models p$ such that $\psi\left(b_{i}, d_{s}\right)$ holds iff $i \in s$.

Proof The proofs of (1) implies (2) and (2) implies (3) are easy. Assume (3), and show (1). We can find $a \models p$ and $A$-mutually indiscernible sequences $\left\langle\left. I_{i}|i<| T\right|^{+}+|A|^{+}\right\rangle$such that for all $i, I_{i}$ is not indiscernible over $A a$. We may assume that the order type of these sequences is $\omega$. The fact that $I_{i}$ is not indiscernible over $A a$ is witnessed by some formula over $A$ and increasing tuples from $I_{i}$, so we may assume that for infinitely many $i$, the formula is the same, and the position of these tuples does not depend on $i$ (maybe changing $a$ ). Then, by Ramsey and compactness, we may assume that $\left\langle I_{i} \mid i<\omega\right\rangle$ is indiscernible over Aa.

(5) follows from (1): Denote $I_{i}=\left\langle a_{i, j} \mid j<\omega\right\rangle$. There is a formula $\psi(x, y)$ over $A$ and an increasing tuple $k_{0}<\cdots<k_{n-1}<r_{0}<\cdots<r_{n-1}$ such that, letting $a_{i, \bar{k}}=\left(a_{i, k_{0}}, \ldots, a_{i, k_{n-1}}\right)$ (and similarly we define $\left.a_{i, \bar{r}}\right), \psi\left(a_{i, \bar{k}}, a\right) \wedge \neg \psi\left(a_{i, \bar{r}}, a\right)$ holds for all $i<\omega$. The sequence $\left\langle b_{i} \mid i<\omega\right\rangle$ defined by $b_{i}=a_{i, \bar{k}}$ when $i$ is even and $b_{i}=b_{i, \bar{r}}$ when $i$ is odd satisfies (5). The fact that $\psi$ is over $A$ is no problem-we can add the parameters to $b_{i}$.

(2) follows from (5) is easy by compactness.

(6) is equivalent to (4) and (7) is equivalent to (5) by a standard application of Ramsey.

(6) follows from (5): By indiscernibility, we may extend the family $\left\langle b_{i} \mid i<\omega\right\rangle$ to $\left\langle b_{r} \mid r \in \mathcal{P}(\omega)\right\rangle$ (with some ordering), and so, for every subset $s \subseteq \mathcal{P}(\omega)$, there is some $d_{s} \models p$ such that $\psi\left(b_{r}, d_{s}\right)$ iff $r \in s$. For $i<\omega$, let $d_{i}=d_{\{r \subseteq \omega: i \in r\}}$. Then for each subset $r \subseteq \omega, \psi\left(b_{r}, d_{i}\right)$ iff $i \in r$. This gives us (6). The same exact argument gives that (7) follows from (4).

Proposition 2.2 If $p$ is a dependent type over $A$, then there is $B \subseteq A$ of size $|B| \leq|T|$ such that $\left.p\right|_{B}$ is dependent.

Proof By Claim 2.1(6), it cannot be that there exists a formula $\varphi(x, y)$ and a set $\left\{a_{i} \mid i<\omega\right\}$ of realizations of $p$ such that for each $s \subseteq \omega$, there is some $c_{s}$ such that $\varphi\left(a_{i}, c_{s}\right)$ holds iff $i \in s$. By compactness, there is no formula $\varphi(x, y)$ such that for all finite $B \subseteq A$ we can find such a set $\left\{a_{i} \mid i<\omega\right\}$ of realizations of $\left.p\right|_{B}$ and such $c_{s}$ for $s \subseteq \omega$. So for each formula $\varphi(x, y)$, there is some finite $B_{\varphi} \subseteq A$ such that there is no such set. Let $B=\bigcup_{\varphi} B_{\varphi}$. Then $\left.p\right|_{B}$ is easily seen to be dependent.

Corollary 2.3 The following are equivalent for a type $p(x)$ over $A$ :

1. $p(x)$ is independent;

2. $\operatorname{rk}-\mathrm{dp}(p) \geq|T|^{+}$. 
Proof If $p$ is dependent, then there is some $B \subseteq A$ such that $\left.p\right|_{B}$ is dependent and $|B| \leq|T|$. By Claim 2.1(3), this means that $\operatorname{rk}-\operatorname{dp}\left(\left.p\right|_{B}\right)<|T|^{+}$, so $\operatorname{rk}-\operatorname{dp}(p)<|T|^{+}$.

In this section we show that some useful properties that are true in dependent theories are actually true in the local context as well.

Fact 2.4 ([3, Theorem 4.11]) If $p$ is a dependent type over $A$, and $a_{i} \vDash p$ for $i<n<\omega$, then $\operatorname{tp}\left(a_{0}, \ldots, a_{n-1} / A\right)$ is also dependent.

Recall the notions of forking and dividing. All the definitions and properties we need can be found in Chernikov and Kaplan [2].

Proposition 2.5 If $p$ is dependent type over a model $M$, and $q$ is a global nonforking extension of $p$ (i.e., an extension to $(\mathfrak{)})$, then $q$ is invariant over $M$.

Proof Suppose that $\varphi\left(x, c_{0}\right) \wedge \neg \varphi\left(x, c_{1}\right) \in q$, where $c_{0} \equiv_{M} c_{1}$. Then using a standard technique, we can assume that $c_{0}, c_{1}$ start an indiscernible sequence $\left\langle c_{0}, c_{1}, \ldots\right\rangle$ over $M$. The set

$$
p(x) \cup\left\{\varphi\left(x, c_{i}\right)^{(i \text { is even })} \mid i<\omega\right\}
$$

is inconsistent by Claim 2.1. This means that for some formula $\psi(x) \in p$,

$$
\left\{\psi(x) \wedge \varphi\left(x, c_{2 i}\right) \wedge \neg \varphi\left(x, c_{2 i+1}\right) \mid i<\omega\right\}
$$

is inconsistent, and so $\psi(x) \wedge \varphi\left(x, c_{0}\right) \wedge \neg \varphi\left(x, c_{1}\right)$ divides over $M$, a contradiction.

Proposition 2.6 (Shrinking of indiscernibles) Suppose that $p(x)$ is a dependent type over $A$ and that $B$ is a set of realizations of $p$.

If $I=\left\langle\left. a_{i}|i<| T\right|^{+}+|B|^{+}\right\rangle$is an A-indiscernible sequence, then some end segment is indiscernible over $A B$. Note that the size of $A$ and the size of the tuple $a_{i}$ do not matter.

Proof We may assume that $B$ is finite. The type $\operatorname{tp}(B / A)$ is dependent by Fact 2.4 . The proof easily follows from Corollary 2.3.

\subsection{Proof of Main Theorem A}

Definition 2.7 Let $p(x)$ be a type over $A$. We say that $p$ is 1-independent over $A$ if there is a realization $a \models p$ and $A$-mutually indiscernible sequences $\left\langle I_{i} \mid i<\omega\right\rangle$ of singletons such that the sequence $\left\langle I_{i} \mid i<\omega\right\rangle$ is indiscernible over $A a$ and for each $i<\omega, I_{i}$ is not indiscernible over $A a$.

We say that $p$ is 1-dependent over $A$ if it is not 1-independent over $A$. We say that $p$ is 1-dependent if it is 1-dependent over any $A^{\prime} \supseteq A$ such that $A^{\prime} \backslash A$ is finite.

Observe that by Claim 2.1, if $p(x)$ is dependent, then it is 1-dependent. Also, as in Remark 1.2, this definition does not depend on $A$.

Claim 2.8 If $p(x)$ is a type over $A$ which is 1-dependent, then

- for every $A^{\prime} \supseteq A$ such that $A^{\prime} \backslash A$ is finite, every $A^{\prime}$-indiscernible sequence

$$
\left\langle\left. a_{i}|i<| T\right|^{+}+|A|^{+}\right\rangle
$$

of tuples satisfying $p$ and singleton $c$, there is some $\alpha<|T|^{+}+|A|^{+}$such that the end segment $\left\langle a_{i} \mid \alpha<i\right\rangle$ is indiscernible over $A^{\prime} c$. 
Proof To simplify notation, assume $A=A^{\prime}=\emptyset$. Toward a contradiction we find a formula $\varphi(\bar{x}, y)$ and an indiscernible sequence $\left\langle\bar{a}_{i} \mid i<\omega\right\rangle$ such that $\bar{a}_{i}$ is a tuple of length $n$ of tuples satisfying $p$ and $\varphi\left(\bar{a}_{i}, c\right)$ holds iff $i$ is even. By the proof of Claim 2.1 (namely, (5) implies (4), with $p=\operatorname{tp}(c)$ ), there is an indiscernible sequence $\left\langle c_{\bar{i}} \mid \bar{i} \in \omega^{n+1}\right\rangle$ (ordered lexicographically) of singletons such that $\varphi\left(\bar{a}_{0}, c_{\bar{i}}\right)$ holds iff the last number in $\bar{i}$ is even. We may also assume (by Ramsey) that the sequence $\left\langle\bar{c}_{\bar{i}} \mid \bar{i} \in \omega^{n}\right\rangle$ is indiscernible over $\bar{a}_{0}$, where $\bar{c}_{\bar{i}}=\left\langle c_{\bar{i}-j} \mid j<\omega\right\rangle$.

Suppose $\bar{a}_{0}=\left(a_{0,0}, \ldots, a_{0, n-1}\right)$, where $a_{0, i} \vDash p$. Since $p$ is 1-dependent over $\emptyset$, there is some $i_{0}<\omega$ such that $\left\langle c_{i_{0}-\bar{i}} \mid \bar{i} \in \omega^{n}\right\rangle$ is indiscernible over $a_{0,0}$. By assumption, $p$ is 1 -dependent over $a_{0,0}$. Inductively, we can find $i_{1}, \ldots, i_{n-1}<\omega$ such that $\bar{c}_{\left(i_{0}, \ldots, i_{n-1}\right)}$ is indiscernible over $\bar{a}_{0}$, a contradiction.

The following theorem implies Main Theorem A.

Theorem 2.9 If $p(x)$ is a type over A which satisfies the conclusion of Claim 2.8, then it is dependent.

Proof Again, assume $A=\emptyset$. Suppose that $p$ is a counterexample. By Claim 2.1, there is an indiscernible sequence $\left\langle\left. a_{i}|i<| T\right|^{+}\right\rangle$such that $a_{i} \models p$, a formula $\varphi(x, y)$, and some tuple $c=\left(c_{0}, \ldots, c_{n-1}\right)$ such that $\varphi\left(a_{i}, c\right)$ holds iff $i$ is even. By assumption, there is some end segment which is indiscernible over $c_{0}$. Applying the conclusion of Claim 2.8 again with $A^{\prime}=\left\{c_{0}\right\}$, we get an end segment which is indiscernible over $c_{0} c_{1}$. Continuing like this, we get an end segment which is indiscernible over $c$, a contradiction.

Since dependent implies 1-dependent, we get the following.

Corollary 2.10 The type $p(x)$ is 1-dependent if and only if it is dependent if and only if it satisfies the conclusion of Claim 2.8.

Corollary 1.5 follows.

Corollary 2.11 A theory $T$ is dependent if and only if for every indiscernible sequence of singletons

$$
\left\langle\left. a_{i}|i<| T\right|^{+}\right\rangle
$$

over some finite A, and for every singleton $c$, there is $\alpha<|T|^{+}$such that $\left\langle a_{i} \mid \alpha<i\right\rangle$ is indiscernible over Ac.

Proof Apply Corollary 2.10 with $p(x)=\{x=x\}$.

\section{Proof of Main Theorem B}

3.1 Preliminaries on $\mathbf{N T P}_{2}$-theories From here up to the end of the section, we assume that the theory is $\mathrm{NTP}_{2}$.

In the study of forking in $\mathrm{NTP}_{2}$-theories, it is sometimes useful to consider independence relations. For instance, we denote $a \downarrow_{B}^{f} C$ for $\operatorname{tp}(a / B C)$ does not fork over $B$. Similarly, $a \downarrow_{B}^{i} C$ means that there is a global extension (i.e., an extension to $(5)$ of $\operatorname{tp}(a / B C)$ which is Lascar invariant over $B$, meaning that if $d$ and $c$ have the same Lascar strong type over $B$, then either both $\varphi(x, c)$ and $\varphi(x, d)$ are in this extension or neither of them is. We do not really need Lascar strong type in this section, because we only work over models. Over a model, Lascar invariance is the same as invariance. 
In the proofs we shall only use the following facts about $\mathrm{NTP}_{2}$-theories. These were proved in [2].

Definition 3.1 (Strict invariance) We say that $\operatorname{tp}(a / B b)$ is strictly invariant over $B$ (denoted by $a \downarrow_{B}^{\text {ist }} b$ ) if there is a global extension $p$, which is Lascar invariant over $B$ (so $a \downarrow_{B}^{i} b$ ) and for any $C \supseteq B b$, if $\left.c \models p\right|_{C}$, then $C \downarrow_{B}^{f} c$.

Fact 3.2 In $\mathrm{NTP}_{2}$-theories,

1. forking equals dividing over models;

2. "Kim's lemma": If $\varphi(x, a)$ divides over $A$, and $\left\langle b_{i} \mid i<\omega\right\rangle$ is a sequence satisfying $b_{i} \equiv_{A} a$ and $b_{i} \downarrow_{A}^{\text {ist }} b_{<i}$, then $\left\{\varphi\left(x, b_{i}\right) \mid i<\omega\right\}$ is inconsistent. In particular, if $\left\langle b_{i} \mid i<\omega\right\rangle$ is an indiscernible sequence, then it witnesses dividing of $\varphi(x, a)$.

Recall the following.

Definition 3.3 Suppose that $p$ is a global type which is invariant over a set $A$.

1. We say that a sequence $\left\langle a_{i} \mid i<\alpha\right\rangle$ is a Morley sequence of a type $p$ over $B \supseteq A$ if $\left.a_{0} \models p\right|_{B}$ and for all $i<\alpha,\left.a_{i} \models p\right|_{B a_{<i}}$. This is an indiscernible sequence over $B$.

2. We let the type $p^{(\alpha)}$ be the union of $\operatorname{tp}\left(\left\langle a_{i} \mid i<\alpha\right\rangle / B\right)$ running over all $B \supseteq A$. This is again an $A$-invariant type.

3. If $q$ is also an $A$-invariant global type, we define $p \otimes q$ as the union of $\operatorname{tp}(a, b / B)$ running over all $B \supseteq A$ where $\left.a \models p\right|_{B}$ and $\left.b \models q\right|_{B a}$. This is also an $A$-invariant global type.

4. Similarly, given a sequence $\left\langle p_{i} \mid i<\alpha\right\rangle$ of $A$-invariant global types, we define $\bigotimes_{i<\alpha} p_{i}$ as the union of $\operatorname{tp}\left(\left\langle a_{i} \mid i<\alpha\right\rangle / B\right)$ running over all $B \supseteq A$, where $\left.a_{i} \models p_{i}\right|_{B a_{<i}}$. Again, this is an $A$-invariant global type.

In the definition above, all types may have infinitely many variables.

Remark 3.4 If $\left\{J_{0}, \ldots, J_{k}\right\}$ is a set of mutually indiscernible sequences over $C \supseteq A$, and $\left\langle a_{i} \mid i<\alpha\right\rangle$ is a Morley sequence of a global $A$-invariant type over $\left\{J_{0}, \ldots, J_{k}\right\} \cup C$, then $\left\{J_{0}, \ldots, J_{k},\left\langle a_{i} \mid i<\alpha\right\rangle\right\}$ is mutually indiscernible over $C$.

(Why? On the one hand, $\left\{J_{0}, \ldots, J_{k}\right\}$ is mutually indiscernible over $C \cup\left\{a_{i} \mid\right.$ $i<\alpha\}$ since $\operatorname{tp}\left(\left\langle a_{i} \mid i<\alpha\right\rangle /\left\{J_{0}, \ldots, J_{k}\right\} \cup C\right)$ does not split over $A$. On the other hand, $\left\langle a_{i} \mid i<\alpha\right\rangle$ is a Morley sequence over $\left\{J_{0}, \ldots, J_{k}\right\} \cup C$ and as such is indiscernible over that set.)

We also need to recall the notions of heir and coheir.

Definition 3.5 A global type $p(x)$ is called a coheir over a set $A$ if it is finitely satisfiable in $A$. Note that in this case, it is invariant over $A$, and $p^{(\alpha)}$ is also a coheir over $A$.

It is called an heir over $A$ if for every formula over $A, \varphi(x, b) \in p$, there exists some $a^{\prime} \in A$ such that $\varphi\left(x, a^{\prime}\right) \in p$.

Claim 3.6 If $p$ is an A-invariant global type, and $p^{(\omega)}$ is both an heir and a coheir over $A$, then any Morley sequence of $p$ over $A,\left\langle a_{i} \mid i<\omega\right\rangle$ satisfies $a_{\geq i} \downarrow_{A}^{\text {ist }} a_{<i}$ for any $i<\omega$.

Proof The type $p^{(\omega)}$ is a global $A$-invariant (so also $A$-Lascar invariant) type that extends $\operatorname{tp}\left(a_{\geq i} / A a_{<i}\right)$, and if $c \models p^{(\omega)} \mid A C$, then $\operatorname{tp}(C / A c)$ is finitely satisfiable over $A$ (since $p^{(\omega)}$ is an heir over $A$ ), and it follows that $C \downarrow_{A}^{f} c$. 
Claim 3.7 Given any global type $p(x)$ and a set $A$, we can find a model $M \supseteq A$ such that $p$ is an heir over $M$.

Proof Construct inductively a sequence of models $M_{i}$ for $i<\omega$. Let $M_{0}$ be any model containing $A$. Let $M_{i+1} \supseteq M_{i}$ be such that for every formula $\varphi(x, y)$ over $M_{i}$, if $\varphi(x, a) \in p$, then there is some such $a$ in $M_{i+1}$. Finally, let $M=\bigcup_{i<\omega} M_{i}$.

Lemma 3.8 Let $M$ be a model. Suppose that $p$ is an $M$-invariant global type such that $p^{(\omega)}$ is an heir-coheir over M. Suppose that I is an endless Morley sequence of $p$ over $M$. If I is indiscernible over $M a$, then $\operatorname{tp}(a / M I)$ does not fork over $M$.

Proof By Fact 3.2, it is enough to see that the type does not divide over $M$. Suppose that $\varphi\left(x, b_{0}\right) \in \operatorname{tp}(a / M I)$ divides over $M$, where $\varphi$ is over $M$ and $b_{0} \subseteq I$. For $i \geq 1$ choose tuples $b_{i} \subseteq I$ of the same length as $b_{0}$ that appear after $b_{0}$ in increasing order. By Claim 3.6, $b_{i} \downarrow_{M}^{\text {ist }} b_{<i}$ so by "Kim's lemma" (Fact 3.2), it must witness dividing. But this is a contradiction to the fact that $I$ is indiscernible over $M a$.

3.2 Proof of the main theorem The following is the key definition in the proof.

\section{Definition 3.9 Suppose that}

1. $p$ is a global $A$-invariant type such that $\left.p\right|_{A}$ is dependent (we call such types $A$-invariant and $A$-dependent);

2. $B$ is some set containing $A$;

3. $\varphi(x, y)$ is a formula over $A$;

4. $a$ is a tuple of length $\lg (y)$.

Then we define alt $(\varphi, B, a, p)$ to be the maximal number $n$ such that there is a realization $\left.\left\langle a_{i} \mid i<n\right\rangle \models p^{(n)}\right|_{B}$, such that $\varphi\left(a_{i}, a\right)$ alternates for $i<n$, that is, such that $\varphi\left(a_{i}, a\right) \Leftrightarrow \neg \varphi\left(a_{i+1}, a\right)$ for $i<n-1$.

Note that alt $(\varphi, B, a, p)$ exists by Claim 2.1(4). Observe also that if $B^{\prime} \supseteq B \supseteq A$, then $\operatorname{alt}(\varphi, B, a, p) \geq \operatorname{alt}\left(\varphi, B^{\prime}, a, p\right)$, but not necessarily the other way. Sometimes we have the following equality.

Lemma 3.10 Suppose that $p$ is a global A-invariant and A-dependent type, $a$ is some tuple, and I is an indiscernible sequence over Aa.

Then for every infinite subset $I^{\prime} \subseteq I$ and for any formula $\varphi(x, y)$ over $A$, $\operatorname{alt}(\varphi, I A, a, p)=\operatorname{alt}\left(\varphi, I^{\prime} A, a, p\right)$.

Proof Obviously, $\operatorname{alt}(\varphi, I A, a, p) \leq \operatorname{alt}\left(\varphi, I^{\prime} A, a, p\right)$.

Conversely, suppose that we have some $n$ such that $\bar{a}=\left.\left\langle a_{i} \mid i<n\right\rangle \models p^{(n)}\right|_{I^{\prime} A}$ alternates as in the definition. Let $\bar{x}=\left(x_{0}, \ldots, x_{n-1}\right)$. We want to show that the type

is consistent.

$$
\left.p^{(n)}(\bar{x})\right|_{I A} \cup\left\{\varphi\left(x_{i}, a\right)^{\left(\text {if } \varphi\left(a_{i}, a\right)\right)} \mid i<n\right\}
$$

Take any finite subset, and write it as $\psi(\bar{x}, b, c) \wedge \xi(\bar{x}, a)$, where $b \subseteq I, c \subseteq A$. As $I^{\prime}$ is infinite and $I$ is indiscernible over $A a$, we can find $b^{\prime} \in I^{\prime}$ such that $b^{\prime} \equiv A a b$, so $\mathfrak{C} \models \exists \bar{x} \psi\left(\bar{x}, b^{\prime}, c\right) \wedge \xi(\bar{x}, a)$ iff $\mathfrak{C} \models \exists \bar{x} \psi(\bar{x}, b, c) \wedge \xi(\bar{x}, a)$. Now, $\psi(\bar{x}, b, c) \in p^{(n)}$, and $p^{(n)}$ is $A$-invariant; hence $\psi\left(\bar{x}, b^{\prime}, c\right) \in p^{(n)}$, and since $\bar{a}$ satisfies $\psi\left(\bar{x}, b^{\prime}, c\right) \wedge \xi(\bar{x}, a)$, we are done.

We will deduce Main Theorem B from the following theorem. 
Theorem 3.11 Suppose that $p(x)$ is a dependent type over $C$ with $\operatorname{rk}-\operatorname{dp}(p) \geq \kappa$. Assume that this is witnessed by $c \models p$ and $\left\{I_{i} \mid i<\kappa\right\}$, where $I_{i}$ has order type $\omega$ for $i<\kappa$.

Then there are

- $C^{\prime} \supseteq C$ with $\left|C^{\prime} \backslash C\right|$ finite, $c^{\prime} \models p$ and $J_{0}$

such that

- $\left\{J_{0}\right\} \cup\left\{I_{i} \mid 0<i<\kappa\right\}$ is mutually indiscernible over $C^{\prime} ; c^{\prime} \equiv C \cup\left\{I_{i} \mid 0<i<\kappa\right\} c$; $J_{0}$ is not indiscernible over $C^{\prime} c^{\prime}$; and

- all the tuples in $J_{0}$ satisfy $p$.

Proof Denote $I_{i}=\left\langle f_{i, j} \mid j<\omega\right\rangle$. By compactness, we can find $f_{i, j}$ for $j \in \mathbb{Z}$ and $i<\kappa$ such that, letting $I_{i}^{\prime}=\left\langle f_{i, j} \mid j \in \mathbb{Z}, j<0\right\rangle,\left\{I_{i}^{\prime} \frown I_{i} \mid i<\kappa\right\}$ is mutually indiscernible over $C$. Let $U$ be a nonprincipal ultrafilter on $\omega$. For $i<\kappa$, let $p_{i}$ be global coheir over $I_{i}^{\prime}$ defined by the following:

- for a formula $\psi(z, y)$ and tuple $a \in \mathfrak{C}, \psi(z, a) \in p_{i}$ iff $\{n<\omega \|$ $\left.\psi\left(f_{i,-n}, a\right)\right\} \in U$.

So each $p_{i}$ is invariant over $\bigcup_{i<\kappa} I_{i}^{\prime}$, and we can consider the type $\left(\bigotimes_{0<i<\kappa} p_{i}^{(\omega)}\right)^{(\omega)}$ and find a model $M \supseteq C \cup \bigcup_{i<\kappa}\left(I_{i}^{\prime} \frown I_{i}\right)$ such that this type is an heir over $M$ (using Claim 3.7).

Let $\left.\left\langle K_{i} \mid i<\kappa\right\rangle \models \bigotimes_{i<\kappa} p_{i}^{(\omega)}\right|_{M}$; then

- each $K_{i}$ is a Morley sequence of $p_{i}$ over $M$,

- since $\left\{I_{i}^{\prime} \frown I_{i} \mid i<\kappa\right\}$ is mutually indiscernible over $C$, and $p_{i}$ is finitely satisfiable in $I_{i}^{\prime},\left\langle K_{i} \mid i<\kappa\right\rangle \equiv_{C}\left\langle I_{i} \mid i<\kappa\right\rangle$ (this follows from the fact that the order type of $I_{i}^{\prime}$ is $\omega^{*}-\omega$ in reverse), and

- by Remark 3.4, $\left\{K_{i} \mid i<\kappa\right\}$ is mutually indiscernible over $M$.

By the second bullet, there is an automorphism of $\mathbb{C}$ that fixes $C$ (but may move $M$ and $p_{i}$ ) and maps $\left\langle K_{i} \mid i<\kappa\right\rangle$ to $\left\langle I_{i} \mid i<\kappa\right\rangle$. By applying it we may assume that $\left\langle K_{i} \mid i<\kappa\right\rangle=\left\langle I_{i} \mid i<\kappa\right\rangle$.

Let $\mu=|T|^{+}$. Let $J=\left\langle d_{i} \mid i<\mu\right\rangle$ be a Morley sequence of $\left(\bigotimes_{0<i<\kappa} p_{i}^{(\omega)}\right)$ over $M I_{0}$ so that $d_{0}$ is the infinite tuple $\left\langle I_{i} \mid 0<i<\kappa\right\rangle$. Note that $I_{0}$ is indiscernible over $J M, J$ is indiscernible over $I_{0} M$, and $\left\{I_{i} \mid i<\kappa\right\}$ is mutually indiscernible over $M \cup\left\{d_{i} \mid 0<i<\mu\right\}$ (by Remark 3.4).

Now, $I_{0}$ is not indiscernible over $C c$. So there are increasing tuples $a_{0}$ and $a_{1}$ from $I_{0}$ of the same length and a formula $\varphi(x, y)$ over $C$ such that $\neg \varphi\left(c, a_{0}\right) \wedge \varphi\left(c, a_{1}\right)$ holds. By indiscernibility, there is an automorphism $\sigma$ of $\mathfrak{c}$ that fixes $J M$ and takes $a_{0}$ to $a_{1}$. Let $c_{0}=c$ and $c_{1}=\sigma\left(c_{0}\right)$. Then $\varphi\left(c_{0}, a_{1}\right) \wedge \neg \varphi\left(c_{1}, a_{1}\right)$ holds.

By Proposition 2.6, for some $\alpha<\mu$, the sequence $J^{\prime}=\left\langle d_{i} \mid \alpha<i<\mu\right\rangle$ is indiscernible over $M c_{0} c_{1}$. By Lemma 3.8, $c_{0} c_{1} \downarrow_{M}^{f} J^{\prime}$.

Let $r(x)$ be a global nonforking (over $M$ ) extension of $\operatorname{tp}\left(c_{0} / M J^{\prime}\right.$ ) (which equals $\left.\operatorname{tp}\left(c_{1} / M J^{\prime}\right)\right)$. Since $\operatorname{tp}\left(c_{0} / C\right)$ is dependent, $r(x)$ is $M$-dependent and $M$-invariant (by Proposition 2.5). Let $n=\operatorname{alt}\left(\varphi, M J, a_{1}, r\right)$, and let $\left\langle e_{i} \mid i<\omega\right\rangle$ be a Morley sequence of $r$ over $M J$ that witnesses this, that is, such that $\varphi\left(e_{i}, a_{1}\right)$ alternates for $i<n$. Let $\left\langle e_{i} \mid \omega \leq i<\omega+\omega\right\rangle$ be a Morley sequence of $r$ over $M J c_{0} c_{1} e_{<\omega}$. 
So

- $I_{0}^{\prime}=\left\langle e_{i} \mid i<\omega+\omega\right\rangle$ is an $M J$-indiscernible sequence, and moreover the union $\left\{I_{i} \mid 0<i<\kappa\right\} \cup\left\{I_{0}^{\prime}\right\}$ is a set of $M J^{\prime}$-mutually indiscernible sequences.

Now, both $\left\langle c_{0}, e_{\omega}, e_{\omega+1}, \ldots\right\rangle,\left\langle c_{1}, e_{\omega}, e_{\omega+1}, \ldots\right\rangle$ are Morley sequences of $r$ over $M J^{\prime}$. But if in addition $\left\langle c_{0}, e_{0}, e_{1}, \ldots\right\rangle$ and $\left\langle c_{1}, e_{0}, e_{1}, \ldots\right\rangle$ are also Morley sequences of $r$ over $M J^{\prime}$, then since one of $c_{0}, c_{1}$, adds an alternation of the truth value of $\varphi\left(x, a_{1}\right)$, this is a contradiction to the choice of $e_{i}$ and to Lemma 3.10 (which we can use because $J$ is indiscernible over $a_{1}$, and $J^{\prime}$ is infinite). Let $c^{\prime} \in\left\{c_{0}, c_{1}\right\}$ be such that $\left\langle c^{\prime}, e_{0}, e_{1}, \ldots\right\rangle$ is not a Morley sequence of $r$ over $M J^{\prime}$. Note that $c^{\prime} \equiv_{M J} c$ and that $M J$ contains $C \cup\left\{I_{i} \mid 0<i<\kappa\right\}$.

So, $\left\langle c^{\prime}, e_{0}, \ldots\right\rangle \neq_{M J^{\prime}}\left\langle c^{\prime}, e_{\omega}, \ldots\right\rangle$, and hence the sequence $I_{0}^{\prime}$ is not indiscernible over $c^{\prime} M J^{\prime}$. Let $J_{0}$ be some infinite subset of $I_{0}^{\prime}$ of order type $\omega$ that witnesses this, let $C^{\prime} \supseteq C$ be such that $\left|C^{\prime} \backslash C\right|$ is finite, and let $C^{\prime} \subseteq M J^{\prime}$ so that $J_{0}$ is not indiscernible over $C^{\prime} c^{\prime}$.

It is now easy to check that all conditions are satisfied.

Now let us conclude.

Proof of Main Theorem B Suppose that $p$ is a dependent type over $A$ with rk-dp $(p) \geq \kappa$. Consider the family $\mathcal{F}$ of triples $\left(s, c, J, A^{\prime}\right)$ such that

- $c \models p, s \subseteq \kappa, J=\left\langle I_{i} \mid i<\kappa\right\rangle ; A \subseteq A^{\prime} ; J$ is a sequence of $A^{\prime}$-mutually indiscernible sequences such that for each $i<\kappa, I_{i}$ is not indiscernible over $A^{\prime} c$; all tuples in $I_{i}$ for $i \in s$ realize $p$.

By assumption, $\mathcal{F}$ is not empty. Define the following order relation on these triples:

- $\left(s, c, J, A^{\prime}\right) \leq\left(s^{\prime}, c^{\prime}, J^{\prime}, A^{\prime \prime}\right)$ iff $\left(s \subseteq s^{\prime}, A^{\prime} \subseteq A^{\prime \prime}\right.$, if $i \in s \cup\left(\kappa \backslash s^{\prime}\right)$, then $I_{i}=I_{i}^{\prime}$ and $\left.c^{\prime} \equiv A^{\prime} \cup\left\{I_{i} \mid i \in s \cup\left(\kappa \backslash s^{\prime}\right)\right\} c\right)$.

It is easy to see that by compactness $\mathscr{F}$ satisfies the conditions of Zorn's lemma, so it has a maximal member $\left(s_{0}, c_{0}, J_{0}, A_{0}^{\prime}\right)$. By Theorem 3.11, $s_{0}=\kappa$ and we are done.

\section{Note}

1. After the appearance of this note, Artem Chernikov has removed this assumption; see [1].

\section{References}

[1] Chernikov, A., "Theories without the tree property of the second kind," $A n$ nals of Pure and Applied Logic, vol. 165 (2014), pp. 695-723. MR 3129735. DOI 10.1016/j.apal.2013.10.002. 428

[2] Chernikov, A., and I. Kaplan, "Forking and dividing in $\mathrm{NTP}_{2}$ theories," Journal of Symbolic Logic, vol. 77 (2012), pp. 1-20. MR 2951626. DOI 10.2178/js1/1327068688. 423, 425

[3] Kaplan, I., A. Onshuus, and A. Usvyatsov, "Additivity of the dp-rank," Transactions of the American Mathematical Society, vol. 365 (2013), pp. 5783-804. MR 3091265. DOI 10.1090/S0002-9947-2013-05782-0. 420, 423

[4] Onshuus, A., and A. Usvyatsov, "On dp-minimality, strong dependence and weight," Journal of Symbolic Logic, vol. 76 (2011), pp. 737-58. MR 2849244. DOI 10.2178/js1/1309952519. 420, 421 
[5] Shelah, S., "Strongly dependent theories," to appear in Israel Journal of Mathematics, preprint, arXiv:math/0504197v4 [math.LO]. 420

[6] Simon, P., "On dp-minimal ordered structures," Journal of Symbolic Logic, vol. 76 (2011), pp. 448-60. Zbl 1220.03037. MR 2830411. DOI 10.2178/js1/1305810758. 420

[7] Usvyatsov, A., "On generically stable types in dependent theories," Journal of Symbolic Logic, vol. 74 (2009), pp. 216-50. Zbl 1181.03040. MR 2499428. DOI 10.2178/js1/1231082310. 420

\title{
Acknowledgment
}

The first author's research was supported in part by SFB grant 878 from Universität Münster.

\author{
Kaplan \\ Hebrew University of Jerusalem \\ Einstein Institute of Mathematics \\ Edmond J. Safra Campus, Givat Ram \\ Jerusalem 91904 \\ Israel \\ itay.kaplan@uni-muenster.de \\ https://sites.google.com/site/itay80/ \\ Simon \\ Université Claude Bernard - Lyon 1 \\ Institut Camille Jordan \\ 43 boulevard du 11 novembre 1918 \\ 69622 Villeurbanne Cedex \\ France \\ pierre.simon@normalesup.org \\ www.normalesup.org/ simon
}

